\title{
Take a look at the bright side: Effects of contrast polarity on gaze direction judgments
}

\author{
BetTina OLK \\ Jacobs University Bremen, Bremen, Germany \\ LAWrenCE A. SyMONS \\ Western Washington University, Bellingham, Washington \\ AND \\ Alan Kingstone \\ University of British Columbia, Vancouver, British Columbia, Canada
}

\begin{abstract}
Observers are inaccurate when judging the gaze direction of eyes shown in negative rather than positive polarity. On the basis of this polarity effect, it has been proposed that gaze is perceived as directed from the dark part of the eye. Our experiment investigated whether direction judgments simply follow this heuristic, as has been suggested. Participants judged the gaze direction of eyes shown at different eccentricities in positive or negative polarity. The error pattern revealed that most errors were incorrect "straight" judgments, suggesting that judgments do not merely follow the heuristic "the dark part does the looking." We suggest that gaze judgments are based on the outcome of a competition between gaze direction signals: Whereas luminance cues may indicate that gaze is directed from the dark part of the eye, geometric cues may indicate the opposite. This interpretation is supported by reduced overall error rates, and fewer incorrect "straight" responses, for more extreme gaze directions.
\end{abstract}

The direction of eye gaze is a key element of social communication, enabling rapid nonverbal signals about potentially rewarding or dangerous stimuli in the world (EiblEibesfeldt, 1989). Gaze direction can also be used to direct other people's attention (e.g., Driver et al., 1999; Hietanen, 1999). The findings that newborns look preferentially at the eyes of other faces and that, by 6 months of age, typically developing infants will monitor where someone else is looking (Haith, Bergman, \& Moore, 1977; Symons, Hains, \& Muir, 1998) underline the importance of gaze information to human communication and behavior.

The morphology of human eyes points toward potentially important factors for the determination of gaze direction (Kobayashi \& Kohshima, 1997). In human eyes, a dark iris is surrounded by a bright sclera; the salient contrast between these two areas should facilitate the decoding of gaze direction. For instance, Ando (2002) showed, by darkening one side of the sclera without shifting the position of the iris, that perception of gaze direction is influenced by the luminance of the sclera. Participants in that study indicated the average of the directions of two eyes using a rod that could be rotated, showing an illusory shift in gaze direction toward the darker side. In another study, Ando (2004) darkened or brightened the area surrounding the eye and reported that gaze seemed to be di- rected toward the side on which the luminance ratio of the sclera to the area surrounding the eye was smaller.

Two further important studies demonstrated the impact of contrast polarity on gaze direction judgments. Ricciardelli, Baylis, and Driver (2000) asked observers to judge whether the gaze of a digitized face was directed straight at the observer or to the observer's left or right. Eyes were presented in either a positive (dark iris on bright sclera) or negative (bright iris on dark sclera) contrast polarity within a face shown in positive contrast polarity (see, e.g., our Figures 1A and 1B). The accuracy of gaze direction judgments was reduced for eyes shown in negative relative to positive contrast polarity. After ruling out that this effect might be due to a mismatch between eye and head polarities or to the unusual look of the eyes when they were shown in negative polarity, the authors suggested that their data can be best explained by an "expert" system for gaze perception, which treats the darker region of the eye as "the part that does the looking." Precisely how negative contrast polarity impacts this expert system or explains their data was left unexplored.

Importantly, an explanation was forthcoming from Sinha (2000), who reported that reversing contrast polarity reverses the perceived direction of gaze. Participants were shown photos depicting individual faces, and they

B. Olk, b.olk@jacobs-university.de 
A

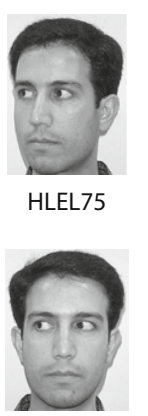

HSEL50

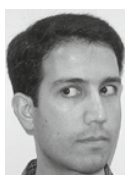

HREL25*

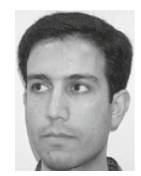

HLEL50

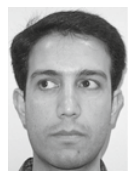

HSEL25*

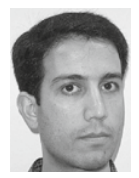

HRES*

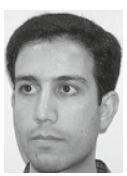

HLEL25*

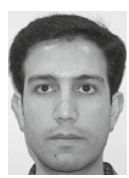

HSES*

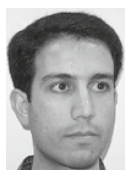

HRER25*

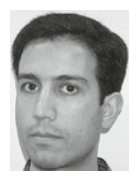

HLES*

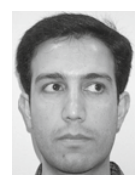

HSER25*

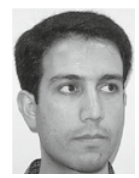

HRER50

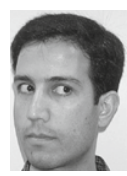

HLER25*

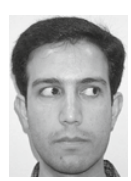

HSER50

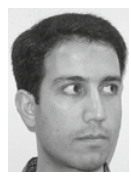

HRER75

B

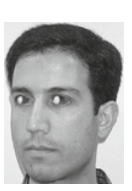

HLEL75

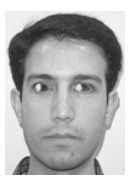

HSEL50

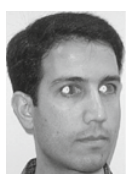

HREL25*

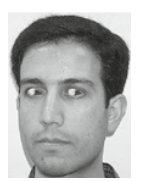

HLEL50

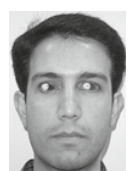

HSEL25*

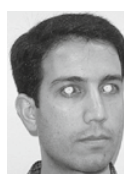

HRES*

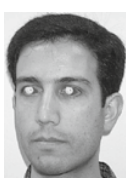

HLEL25*

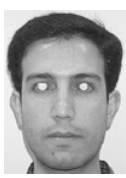

HSES*

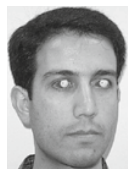

HRER25*

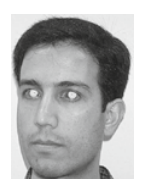

HLES*

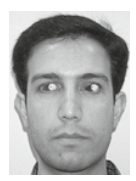

HSER25*

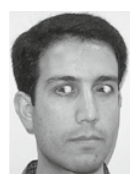

HRER50

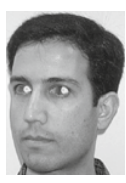

HLER25*

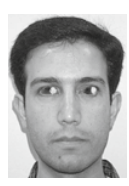

HSER50

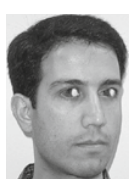

HRER75

Figure 1. (A) Eyes in positive polarity. (B) Eyes in negative polarity. See the text for further details. HL, head left; HS, head straight; HR, head right; EL, eyes left; ES, eyes straight; ER, eyes right. ${ }^{*}$ Conditions also used by Ricciardelli et al. (2000).

judged whether the person was looking to the left or right. Each gaze was presented in positive or in negative polarity. In both conditions, the participants tended to judge gaze in the direction of the dark part of the eye (the dark iris or dark sclera, respectively). Reversing the eye polarity thus led to the reversal of gaze direction judgments. Sinha then suggested that the visual system relies on a heuristic that estimates "which side is dark," and that this heuristic is followed despite the abnormal geometrical properties of eyes shown in negative polarity. Close inspection of the data obtained for the different faces, each of which differed slightly with respect to the eccentricity of eye direction,

suggests that the more extreme the eye direction, the more extreme the reversal effect. These data were drawn from only four pictures, which varied not only in eye direction but also in other dimensions, such as head direction, gender, familiarity, and identity. Thus, one issue deserving further investigation is how the proposed heuristic is applied for polarity-reversal pictures with different gaze directions. We systematically examined this issue in our study, and in keeping with Sinha's observations, we hypothesized that more extreme gaze directions would lead to more rigorous applications of the gaze direction heuristic. In other words, as gaze direction became more extreme, the perceived direction of gaze would show greater reversal when polarity was switched from positive to negative.

One important deviation between Sinha's (2000) investigation and ours concerns the response options made available to participants. Sinha's participants were only permitted to respond that gaze was directed to the left or right. This means that when polarity was reversed, all response errors were consistent with a reversal in the perceived direction of gaze. In contrast, we provided a third response option - eyes straight - in order to obtain a finer-grained analysis of how contrast reversal affects the perception of gaze direction. The importance of this design modification is highlighted by the fact that in one of four experiments by Ricciardelli et al. (2000, Experiment 1), participants were provided with precisely these three response options, and the authors noted that many straight-gaze response errors were committed. Unfortunately, these straight-gaze data were not presented in the report of their study (in the remaining experiments, they obtained only "left/right" response judgments, raising the same potential limitation as in the Sinha study).

Thus, the aims of the present study were twofold. First, we aimed to obtain a finer-grained analysis of gaze perception judgments and the types of errors that are made with negative-polarity images. Second, we sought to examine whether perceived gaze reversals are more pronounced as left/right gaze directions become more extreme. We achieved these goals by recording straight-gaze as well as "left/right" judgments and by varying the eccentricity of gaze direction of the stimuli systematically. Furthermore, we ran our experiment in two ways. For one group, we presented a randomized order of positive- and negativepolarity pictures within the same experimental block. This methodology dovetails with those of Sinha (2000) and Ricciardelli et al. (2000). For a second group, we presented positive- and negative-polarity pictures in separate blocks, reasoning that this would provide participants with the opportunity to adopt a response set to compensate for perceptual reversals - for example, if negative-polarity pictures reverse the correct perception of gaze direction, participants could consistently respond correctly by selecting a response that is the opposite of their actual perception.

\section{METHOD}

\section{Participants}

Two groups of observers, who all gave informed consent, took part and received course credit. Each group consisted of 14 participants (mean age 20 years) with normal or corrected-to-normal vision. 


\section{Stimuli and Procedure}

We closely followed the methodology of Ricciardelli et al. (2000, Experiment 1). This ensures that our stimuli match those of previous experiments, which in turn justifies generalizations across studies. Digitized grayscale photographs of a single person ("the looker") were taken with different head and eye directions. In keeping with Ricciardelli et al. and to increase difficulty, head direction was either $25^{\circ}$ to the left (HL) or to the right (HR) or straight ahead (HS). For each head direction, the eyes were either aligned with the head or averted $25^{\circ}$ or $50^{\circ}$ to the left or right with respect to the head midline. To create averted-head stimuli, the HL stimuli were mirrored using Adobe Photoshop to obtain the HR stimuli. For the HS stimuli, the images with gazes directed to the left were the mirror images of those with gazes directed to the right. These combinations led to gazes either straight at the observer (ES) or averted to the left (EL) or the right (ER) by $25^{\circ}, 50^{\circ}$, or $75^{\circ}$. The complete stimulus set is illustrated in Figure 1.

In half of the trials, the contrast polarity was normal ("positive"), showing eyes with a dark pupil on a bright sclera. In the other half, the contrast polarity was reversed ("negative"), with a bright pupil on a dark sclera. Contrast reversals of the eyes were accomplished using Adobe Photoshop. Care was taken to ensure that the area surrounding the eyes did not merge with the black iris for positivepolarity eyes or with the black sclera for negative-polarity eyes. The pictures were presented on a white background on an 18-in. monitor. The dimensions of the pictures were $18 \mathrm{~cm}$ high $\times 13 \mathrm{~cm}$ wide, and the viewing distance was approximately $40 \mathrm{~cm}$. Each image was presented until response or for a maximum of $1,112 \mathrm{msec}$, whichever came first (a beep occurred at $1,112 \mathrm{msec}$ if no response had been given). Each stimulus combination was shown 25 times, resulting in 750 trials for each participant. The intertrial interval was $500 \mathrm{msec}$, and a break was allowed after 75 trials.

The participants were instructed to indicate the perceived direction of the looker's gaze with respect to themselves. They were provided with the following written instructions:

At the beginning of each trial, an asterisk will be presented on the screen. This will be followed by a photograph of a face. Your task is to decide whether the person's EYES are looking to YOUR LEFT, YOUR RIGHT or STRAIGHT AT YOU. Please indicate your response by pressing the corresponding keys on the computer: $\mathrm{L}=$ to your left; $\mathrm{C}=$ straight at you; $\mathrm{R}=$ to your right. In the photograph, the head may be directed straight ahead, turned to the left or turned to the right. It is important that you indicate only whether the EYES are looking to your left, to your right or straight at you. In addition, in one half of the experiment the photographs are shown with the eyes in positive polarity and in the other half the eyes are in negative polarity. An example of each is provided below. You will only have 1 second to respond on the keyboard, so please respond quickly and accurately.

The response keys on the keyboard were marked with stickers (the "f" key with $L$, the "b" key with $C$, and the "j" key with $R$ ). Participants were provided with 10 example pictures, showing the five stimuli with the head direction to the right, once apiece with the eyes of each stimulus in positive and negative polarity. The correct response was given underneath each picture.

For Group Random, we randomized the order of the positive- and negative-polarity stimuli within the experimental block. This block was preceded by 12 instances of each type of polarity, presented in random order. For Group Block, the positive and negative stimuli were shown in separate blocks, with the order of the two blocks counterbalanced between participants. Each of the experimental blocks was preceded by 12 instances of the polarity appropriate for that block.

\section{RESULTS}

\section{Accuracy}

We analyzed response accuracy to test whether performance in judging the gaze direction of eyes shown in negative polarity is worse than that for eyes shown in positive polarity (the polarity effect). Trials were excluded if no response was given before time-out or if the response time was less than $100 \mathrm{msec}$. The mean percentage of correctly answered trials was averaged across participants and subjected to a repeated measures ANOVA with the independent variables polarity (positive, negative), head direction (3 levels), and gaze direction (5 levels). The data from both groups were first analyzed independently and then compared. The results are illustrated in Figure 2.

Group Random gave more correct responses when judging eyes in positive rather than negative polarity $\left[F(1,13)=24.61, M S_{\mathrm{e}}=2,027.21, p<.001\right]$, showing a clear polarity effect. There were further main effects of head direction $\left[F(2,26)=10.33, M S_{\mathrm{e}}=710.77, p<.002\right]$ and gaze direction $\left[F(4,52)=10.17, M S_{\mathrm{e}}=539.62, p<\right.$ $.001]$. Importantly, the polarity effect was modulated by head and gaze direction, because the two-way interactions

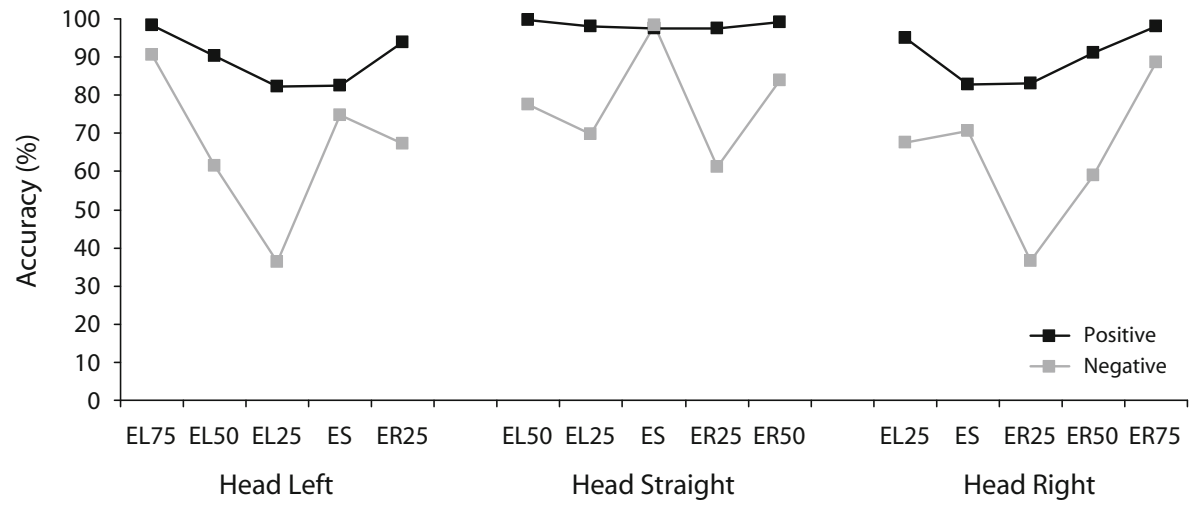

Figure 2. Percentages of correct responses for combinations of three head and five gaze directions, shown separately for eyes in positive and in negative polarity, averaged over Groups Random and Block. EL, eyes left; ES, eyes straight; ER, eyes right. 
polarity $\times$ gaze direction $\left[F(4,52)=4.18, M S_{\mathrm{e}}=186.72\right.$, $p<.006]$ and head direction $\times$ gaze direction $[F(8,104)=$ 7.28, $\left.M S_{\mathrm{e}}=493.74, p<.001\right]$, as well as the three-way interaction polarity $\times$ head direction $\times$ gaze direction $\left[F(8,104)=9.84, M S_{\mathrm{e}}=231.25, p<.001\right]$, all reached significance. The interactions reflect the fact that poorer performance in judging the direction of negative versus positive eyes was particularly apparent when (1) the head was directed straight ahead and gaze direction was averted and (2) the head was averted and eye direction was aligned with head direction. No further effects were significant.

Group Block also showed a polarity effect, with more correct responses for positive than for negative eyes $\left[F(1,13)=20.71, M S_{\mathrm{e}}=2,971.0, p<.002\right]$. This effect was modulated by head and gaze direction [main effect of head direction, $F(2,26)=12.4, M S_{\mathrm{e}}=305.78, p<.001$; main effect of gaze direction, $F(4,52)=9.47, M S_{\mathrm{e}}=326.52$, $p<.001]$. The two-way interactions [polarity $\times$ head direction, $F(2,26)=3.76, M S_{\mathrm{e}}=214.61, p<.04$; polarity $\times$ gaze direction, $F(4,52)=4.35, M S_{\mathrm{e}}=162.89, p<.005$; head direction $\times$ gaze direction, $F(8,104)=6.96, M S_{\mathrm{e}}=$ $393.48, p<.001]$, as well as the three-way interaction polarity $\times$ head direction $\times$ gaze direction $[F(8,104)=6.9$, $\left.M S_{\mathrm{e}}=319.28, p<.001\right]$, reached significance.

The results for Group Block thus replicate those for Group Random. This was confirmed statistically by an ANOVA comparing the performance of Groups Random and Block. The between-participants factor of group had no significant effect and did not interact with any of the other factors.

\section{Error Pattern}

To analyze whether responses reflected the heuristic in which contrast polarity reverses perceived gaze direction, as well as whether this heuristic is applied more as gaze direction becomes more extreme, the percentages of incorrect left-, straight-, and right-gaze responses among all of the responses (correct and incorrect) were determined for each stimulus.

As can be seen in Table 1 and Figure 3, the majority of errors were incorrect "straight" judgments and not incorrect "left" or "right" judgments. Table 1 provides the percentages of incorrect responses separately for each group, whereas Figure 3 illustrates the results averaged over both groups. In a comparison of the responses given to eyes presented in negative versus positive polarity, it is apparent that the increase in error rates for the negative-polarity eyes relative to the positive-polarity ones is due mainly to an increase of erroneous "straight" responses. To analyze whether significantly more straight errors occurred than opposite (left/right) errors, the percentages of incorrect straight-gaze and incorrect opposite responses were subjected to an ANOVA with the factors polarity (positive, negative), response (straight, opposite), and condition (12 levels). ${ }^{1}$ One such analysis was run for each group.

The analyses showed that more straight-gaze than opposite responses were given and that this pattern was modulated by condition and polarity, as indicated by the significant interactions. The analysis for Group Random returned a significant main effect of response $[F(1,13)=$
Table 1

Distribution of Percentages of Incorrect Left, Straight, and Right Responses

\begin{tabular}{|c|c|c|c|c|c|c|}
\hline \multirow[b]{2}{*}{ Stimulus } & \multicolumn{3}{|c|}{ Positive Polarity } & \multicolumn{3}{|c|}{ Negative Polarity } \\
\hline & Left & Straight & Right & Left & Straight & Right \\
\hline \multicolumn{7}{|c|}{ Group Random } \\
\hline HLEL75 & & 1.15 & 0.29 & & 8.91 & 2.87 \\
\hline HLEL50 & & 11.21 & 0.29 & & 24.35 & 8.70 \\
\hline HLEL25 & & 22.70 & 0 & & 66.86 & 4.99 \\
\hline HLES & 1.16 & & 22.54 & 4.57 & & 20.29 \\
\hline HLER25 & 0.29 & 7.76 & & 2.62 & 28.49 & \\
\hline HSEL50 & & 0 & 0 & & 27.95 & 1.44 \\
\hline HSEL25 & & 0.88 & 0.29 & & 25.86 & 1.44 \\
\hline HSES & 0.83 & & 0 & 1.43 & & 0.57 \\
\hline HSER 25 & 0 & 0.57 & & 0 & 39.54 & \\
\hline HSER50 & 0 & 0.86 & & 1.15 & 10.66 & \\
\hline HREL25 & & 4.01 & 0 & & 20.86 & 6.57 \\
\hline HRES & 22.41 & & 1.44 & 25.21 & & 6.59 \\
\hline HRER25 & 0 & 21.49 & & 6.07 & 59.83 & \\
\hline HRER50 & 0.58 & 10.06 & & 11.01 & 27.54 & \\
\hline HRER75 & 0.29 & 1.72 & & 2.31 & 10.66 & \\
\hline \multicolumn{7}{|c|}{ Group Block } \\
\hline HLEL75 & & 2.03 & 0 & & 4.36 & 2.33 \\
\hline HLEL50 & & 7.60 & 0.29 & & 20.12 & 23.62 \\
\hline HLEL25 & & 11.92 & 0.87 & & 43.52 & 12.10 \\
\hline HLES & 4.05 & & 7.23 & 11.30 & & 14.00 \\
\hline HLER25 & 1.17 & 3.23 & & 19.40 & 15.10 & \\
\hline HSEL50 & & 0.58 & 0 & & 4.58 & 10.60 \\
\hline HSEL 25 & & 2.91 & 0 & & 24.21 & 8.65 \\
\hline HSES & 4.05 & & 0.29 & 0.86 & & 0.29 \\
\hline HSER 25 & 0.29 & 4.34 & & 6.94 & 30.92 & \\
\hline HSER50 & 0.58 & 0.29 & & 12.61 & 7.74 & \\
\hline HREL 25 & & 5.19 & 0.86 & & 19.13 & 17.97 \\
\hline HRES & 5.78 & & 4.62 & 13.58 & & 13.29 \\
\hline HRER25 & 0 & 12.43 & & 12.07 & 49.43 & \\
\hline HRER50 & 0 & 6.90 & & 21.16 & 22.61 & \\
\hline HRER75 & 0 & 2.05 & & 4.35 & 5.22 & \\
\hline
\end{tabular}

Note-Empty cells refer to the correct response for a given category. HL, head left; HS, head straight; HR, head right; EL, eyes left; ES, eyes straight; ER, eyes right.

$\left.30.52, M S_{\mathrm{e}}=1,394.31, p<.001\right]$ and the following significant interactions: response $\times$ condition $[F(11,143)=$ 9.23, $\left.M S_{\mathrm{e}}=225.72, p<.001\right]$; response $\times$ polarity $\left[F(1,13)=11.92, M S_{\mathrm{e}}=1,201.23, p<.005\right]$; and response $\times$ condition $\times$ polarity $\left[F(11,143)=4.83, M S_{\mathrm{e}}=\right.$ 125.07, $p<.001]$. As is evident in Table 1 , the extent to which straight errors exceeded opposite errors varied in magnitude, consistent with the fact that the overall error rate varied with changes both to eye polarity and head/ gaze direction. This pattern is also reflected in the significant main effects of condition $[F(11,143)=11.05$, $\left.M S_{\mathrm{e}}=220.19, p<.001\right]$ and polarity $[F(1,13)=22.47$, $\left.M S_{\mathrm{e}}=1302.54, p<.001\right]$, as well as by their interaction $\left[F(11,143)=5.86, M S_{\mathrm{e}}=95.22, p<.001\right]$, confirming our accuracy analysis. For Group Block, there were also more "straight" responses, in particular when the eyes were only averted $25^{\circ}$ to the left or right. This varying effect of response is reflected in the significant key interaction response $\times$ condition $\times$ polarity $[F(11,143)=2.94$, $\left.M S_{\mathrm{e}}=186.09, p<.003\right]$. Furthermore, and as for Group Random, the two-way interactions polarity $\times$ condition $\left[F(11,143)=6.22, M S_{\mathrm{e}}=105.94, p<.001\right]$ and condition $\times$ response $\left[F(11,143)=5.39, M S_{\mathrm{e}}=218.79, p<\right.$ 

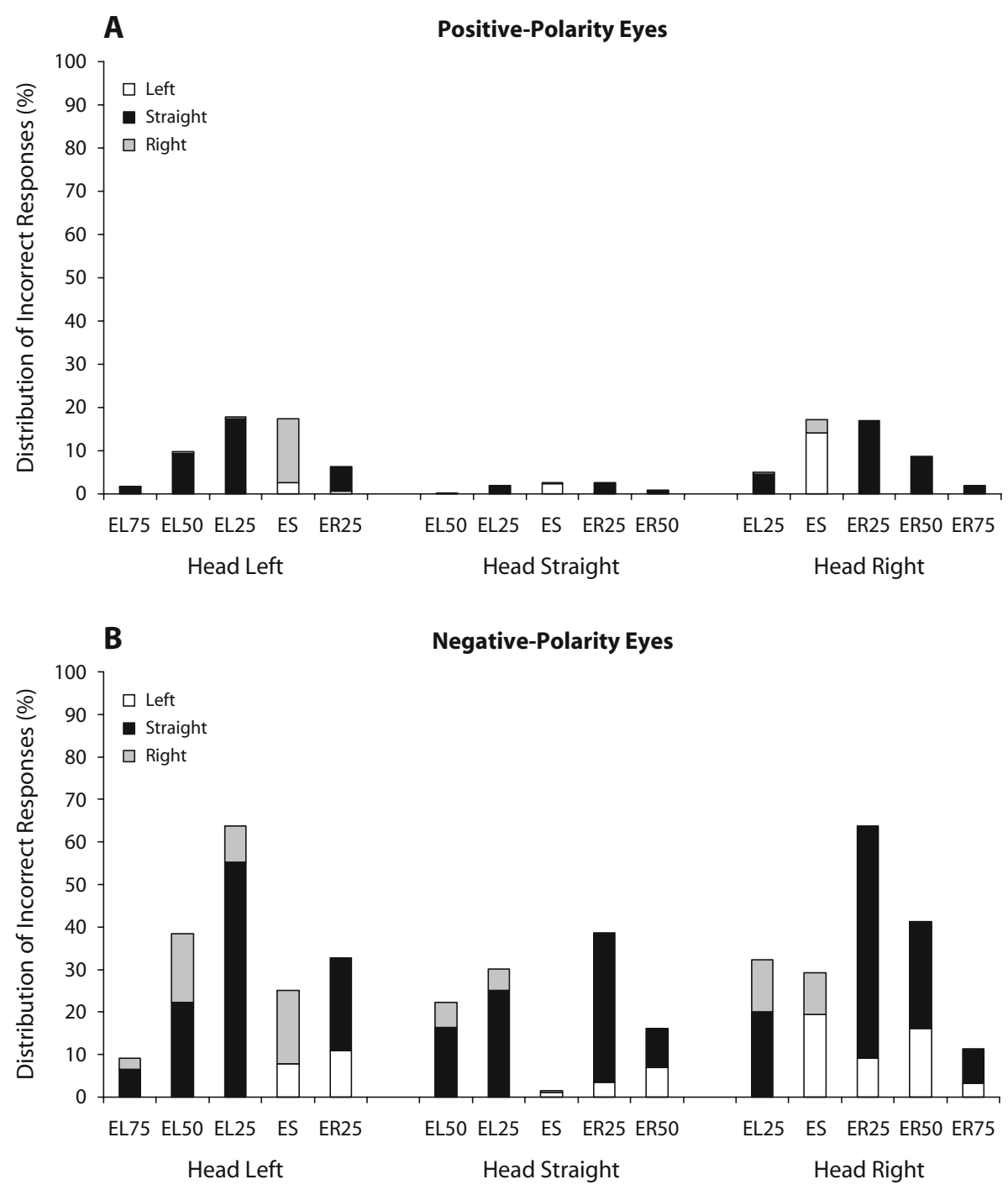

Figure 3. Distributions of incorrect "left," "right," and "straight" responses given for (A) eyes in positive polarity and (B) eyes in negative polarity, averaged over Groups Random and Block. The values represent percentages of incorrect responses out of all responses (correct and incorrect), split over the three response options. EL, eyes left; ES, eyes straight; ER, eyes right.

$.001]$ and the main effects of condition $[F(11,143)=9.74$, $\left.M S_{\mathrm{e}}=162.31, p<.001\right]$ and polarity $[F(11,13)=19.32$ $\left.M S_{\mathrm{e}}=1,702.5, p<.002\right]$ were significant. When comparing the results for both groups with those of an ANOVA with group as a between-participants factor, the key interaction response $\times$ condition $\times$ polarity again reached significance $\left[F(11,286)=6.7, M S_{\mathrm{e}}=155.58, p<.001\right]$ and was not modulated by the between-participants factor group $(F<1)$.

\section{DISCUSSION}

In their studies, Sinha (2000) and Ricciardelli et al. (2000) proposed an expert system for gaze direction judg- ments, which their results revealed in the high amounts of errors that participants made when asked to judge the gaze direction of contrast-reversed eyes. Sinha further suggested that the expert system judges gaze according to the heuristic "which side is dark." The strict application of this heuristic would result in a specific type of error if the contrast of an image were reversed; to be precise, a leftdirected gaze would be perceived as looking to the right, and a right-directed gaze would be perceived as looking to the left. Moreover, as gaze direction became more extreme, so would the perceived reversal in gaze direction.

Although this elegant proposal of a heuristic accounted for both Sinha's (2000) and Ricciardelli et al.'s (2000) data, there were several sound reasons to investigate the 
proposal further. First, Sinha's study had confounded the degree that eye direction was to the left or right with other changes to the images, such as size, gender, familiarity, and head direction of the depicted persons. Therefore, it is possible that any one of these other factors, or their combination, could have modified the reversal effect, rather than the extremeness of gaze. Second, participants were permitted to give only "left/right" responses, so any response error would be, by definition, the opposite of the correct judgment. This implies that the experimental designs might have overestimated the extent to which a response reversal reflected a reversal in the true perception of gaze direction. Third, we noticed that on the one occasion when participants were afforded the opportunity to execute a "straight" response in addition to the "left" and "right" responses (Ricciardelli et al., 2000, Experiment 1), it was observed that many errors were no longer response reversals (although how many "straight" response errors were executed, and under what conditions, was not reported).

The present study therefore presented participants with the opportunity to perform a straight- as well as a left- or right-gaze judgment and systematically varied gaze and head direction, while keeping factors such as the size, familiarity, and gender of the stimuli constant. The results were clear cut: Participants were quite poor at judging gaze direction when presented with contrast-reversed eyes, replicating the basic finding of Sinha (2000) and Ricciardelli et al. (2000). Furthermore, consistent with their interpretation that this effect reflects a perceptual phenomenon driven by an expert system applying an obligatory heuristic, presenting positive- and negative-polarity images to participants in separate blocks (Group Block) rather than mixing both types of images randomly (Group Random) had no reliable impact on performance. It is reasonable to assume that under blocked conditions, observers had the opportunity to strategically change their responses to negative-polarity eyes, but they do not seem to have done so, because performance did not improve for Group Block as compared with Group Random. Of course, it is possible that with unlimited viewing time, observers may well be able to apply a strategy and arrive at correct judgments more frequently. The initial gaze direction judgment, however, seems to be quite impenetrable to strategies.

Interestingly, though, we observed that the majority of responses were incorrect straight-gaze rather than opposite judgments (i.e., "left" for a gaze directed to the right and "right" for a gaze directed to the left). ${ }^{2}$ This finding indicates that reversing contrast polarity does not necessarily reverse the perceived direction of gaze, as it has previously been proposed. We observed that when eyes gazing to the left or right were presented in negative polarity, on most occasions participants would judge, incorrectly, that the gaze was directed straight ahead. This echoes the remark of Ricciardelli et al. (2000) and supports our concern that when provided only with "left/right" response alternatives, the response reversals obtained by Sinha (2000) and in most of the experiments by Ricciardelli et al. overestimate the extent of the perception of gaze in the direction of the dark part of the eye when images are shown in negative polarity. The latter result is further reinforced by our find- ing that as gaze direction becomes more extreme, so that the eyes look farther to the left or right, participants make fewer errors, and especially fewer "left/right" response errors. This finding stands in marked contrast to our prediction - on the basis of the study and heuristic put forward by Sinha - that as gaze direction becomes more extreme, the perceived direction of gaze should show greater reversals (and thus more errors) for negative-polarity images. Thus, our results indicate that the expert system does not simply judge gaze by default as looking in the direction of the dark part of the eye.

Clearly, judgments of gaze direction must involve more than the application of the simple heuristic "the dark part of the eyes does the looking." What other factors might contribute? One of these most certainly is head direction. In line with previous studies, we found that the polarity effect was modulated by head direction (Gamer \& Hecht, 2007; Gibson \& Pick, 1963; Ricciardelli et al., 2000). Performance was especially poor for negative-polarity stimuli when the head was directed straight and gaze direction was averted, as well as when head direction was averted and gaze direction was aligned with the head direction. In addition, since the geometric properties of the eyes vary with head direction, these results illustrate a significant interaction during gaze direction judgments between directional cues based on the geometry of the eyes and those provided by the contrast polarity between the dark part of the eye (e.g., the iris) and the sclera.

A question still to be answered is why most of the errors, especially when negative-polarity eyes were judged, were incorrect straight-gaze judgments. An explanation for the performance decline due to incorrect "straight" judgments could be that gaze direction judgments reflect the outcome of a competition between different gaze direction signals. For negative-polarity eyes, information derived from geometric features, such as the relative size of the sclera on either side of the iris, conflicts with information provided by polarity and luminance cues, and as such more often than not results in intermediate perception of a straight gaze. ${ }^{3}$ Consider, for example, a stimulus such as HRER25, for which participants made many errors and showed frequent incorrect "straight" responses: Geometric features such as the shape of the sclera and the round shape of the iris would indicate that gaze is directed to the right of the observer. The round iris, with a diameter of $1.15^{\circ}$ of visual angle in our stimuli, was clearly located toward the right of the eye (length: $3^{\circ}$ of visual angle). Polarity and luminance, on the other hand, would suggest that gaze is directed to the left of the observer ("which side is dark"). The performance improvement that occurs when gaze is directed very far to the left or right supports this explanation. For such stimuli, geometrical cues may be weighted more than polarity cues (Symons, Lee, Cedrone, \& Nishimura, 2004); as such, the effect of polarity reversal is weaker, and performance improves.

In summary, our results are in line with the proposals that an expert system controls the judging of gaze direction and that this system follows a heuristic in an obligatory fashion, as previously suggested by Sinha (2000) and Ricciardelli et al. (2000). However, with respect to what 
this heuristic entails, we suggest that it includes more than polarity cues - namely, that geometric information is important as well. If polarity cues were the main determinant for gaze direction judgments, response errors for negative-polarity images should have been the reverse of the correct answers-for example, incorrectly judging a left gaze as looking to the right. Our results show, though, that the majority of response errors for negative-polarity images are due to gaze being incorrectly perceived as looking straight ahead. On the basis of these data, we propose that gaze judgments are based on the outcome of a competition between different gaze direction signals. For example, whereas luminance cues may indicate that gaze is directed toward the dark part of the eye, geometric cues may indicate the opposite. Future research should test whether such an (extended) heuristic could also be applied to judgments of gaze in the vertical plane, as well as focusing on the neural underpinnings of the observed results. One candidate region for involvement in the "expert system" may be the superior temporal sulcus. Studies using functional neuroimaging have reported cortical activation in response to gaze direction in temporal areas analogous to the monkey superior temporal sulcus, as well as in parietal cortex (Engell \& Haxby, 2007; Hoffman \& Haxby, 2000; Wicker, Michel, Henaff, \& Decety, 1998). Further evidence for cortical involvement comes from nonhuman primate studies, which have indicated cells in the inferior temporal cortex that respond selectively to faces and eyes (for a review, see Desimone, 1991) and cells in the superior temporal sulcus that respond selectively to particular gaze directions (see, e.g., Perrett et al., 1985).

\section{AUTHOR NOTE}

This research was funded by a fellowship from the Michael Smith Foundation for Health Research awarded to B.O., as well as by grants to A.K. from the Natural Sciences of Engineering Research Council of Canada and the Michael Smith Foundation for Health Research. The authors thank Meena Jassal and Sherief Hammad for assistance with the data collection and stimulus generation. Correspondence related to this article may be sent to B. Olk, School of Humanities and Social Sciences, Jacobs University Bremen, Campus Ring 1, 28759 Bremen, Germany (e-mail: b.olk@jacobs-university.de).

\section{REFERENCES}

ANDo, S. (2002). Luminance-induced shift in the apparent direction of gaze. Perception, 31, 657-674.

ANDo, S. (2004). Perception of gaze direction based on luminance ratio. Perception, 33, 1173-1184.

Desimone, R. (1991). Face-selective cells in the temporal cortex of monkeys. Journal of Cognitive Neuroscience, 3, 1-8.

Driver, J., Davis, G., Ricciardelli, P., Kidd, P., Maxwell, E., \& BARON-CoHEN, S. (1999). Gaze perception triggers reflexive visuospatial orienting. Visual Cognition, 6, 509-540.

Eibl-Eibesfeldt, I. (1989). Human ethology. New York: Aldine de Gruyter.

Engell, A. D., \& Haxby, J. V. (2007). Facial expression and gazedirection in human superior temporal sulcus. Neuropsychologia, $\mathbf{4 5}$, 3234-3241.

Gamer, M., \& Hecht, H. (2007). Are you looking at me? Measuring the cone of gaze. Journal of Experimental Psychology: Human Perception \& Performance, 33, 705-715.
Gibson, J. J., \& PICK, A. D. (1963). Perception of another person's looking behavior. American Journal of Psychology, 76, 386-394.

Haith, M. M., Bergman, T., \& Moore, M. J. (1977). Eye contact and face scanning in early infancy. Science, 198, 853-855.

Hietanen, J. K. (1999). Does your gaze direction and head orientation shift my visual attention? NeuroReport, 10, 3443-3447.

Hoffman, E. A., \& HaXby, J. V. (2000). Distinct representations of eye gaze and identity in the distributed human neural system for face perception. Nature Neuroscience, 3, 80-84.

Kobayashi, H., \& Kohshima, S. (1997). Unique morphology of the human eye. Nature, 387, 767-768.

Perrett, D. I., Smith, P. A., Potter, D. D., Mistlin, A. J., Head, A. S., Milner, A. D., \& JeEves, M. A. (1985). Visual cells in the temporal cortex sensitive to face view and gaze direction. Proceedings of the Royal Society B, 223, 293-317.

Ricciardelli, P., BaYlis, G., \& Driver, J. (2000). The positive and negative of human expertise in gaze perception. Cognition, 77, B1-B14.

SinHa, P. (2000). Last but not least: Here's looking at you, kid. Perception, 29, 1005-1008.

Symons, L. A., Hains, S. M. J., \& Muir, D. W. (1998). Look at me: Fivemonth-old infants' sensitivity to very small deviations in eye-gaze during social interactions. Infant Behavior \& Development, 21, 531-536.

Symons, L. A., Lee, K., Cedrone, C. C., \& Nishimura, M. (2004). What are you looking at? Acuity for triadic eye gaze. Journal of General Psychology, 131, 451-469.

Wicker, B., Michel, F., Henaff, M. A., \& Decety, J. (1998). Brain regions involved in the perception of gaze: A PET study. NeuroImage, 8, 221-227.

\section{NOTES}

1. Because we were interested in errors only, conditions for which the response "straight" was correct (i.e., the HLES, HSES, and HRES conditions) were excluded from the analysis.

2 . The analyses of mean response times for correct and incorrect responses to negative-polarity eyes revealed that the high percentage of incorrect straight-gaze judgments was not due to a speed-accuracy trade-off. For Group Random, incorrect "straight" responses (706 msec) were given significantly more slowly than correct "straight" responses $(620 \mathrm{msec})[t(11)=6.13, p<.001]$ and correct "left/right" responses $(632 \mathrm{msec})[t(11)=3.96, p=.002]$, and they did not differ from incorrect "left/right" responses (694 msec) $[t(11)=0.150, p=.876]$. These results were replicated for Group Block; their incorrect "straight" responses $(631 \mathrm{msec})$ were given more slowly than correct "straight" $(587 \mathrm{msec})[t(11)=3.0, p=.012]$ and correct "left/right" (590 msec) $[t(11)=2.2, p=.048]$ responses and did not differ from incorrect "left/ right" responses $(641 \mathrm{msec})[t(11)=-0.367, p=.720]$. Please note that for each group, the data of 2 participants had to be excluded from the analyses because of a lack of incorrect "left/right" responses.

3. It could be argued that frequent "straight" responses are in line with the previously proposed heuristic "the dark part does the looking." For example, with heads turned to look to the side, negation might yield an eye configuration that corresponds to a straight gaze (e.g., the negativepolarity stimulus HRER 75 corresponds to an eye configuration in which the person is looking straight ahead). Contrast reversal may thus create an image in which the dark part is where the iris would be for straight viewing, and this might affect participants' responses in such conditions. However, for other stimuli, this reasoning seems to work less well. For example, for the contrast-reversed HSER50 stimulus, one might claim that the location of the dark sclera is where the iris would normally be. In this case, many "straight" errors should occur; in fact, more errors generally and more straight errors should be made than during the HSER 25 condition. For the latter condition, fewer "straight" but more "left" responses would be expected on the basis of a contrast heuristic. We found, though, that performance was much worse for HSER25 stimuli and that the number of incorrect "straight" responses in that condition was much larger than for HSER50 stimuli.

(Manuscript received October 1, 2007; revision accepted for publication April 23, 2008.) 